\title{
Studies on Platform-Type Organization Design Based on Leadership Promotion
}

\author{
Xin MA ${ }^{1}$, Shuhui LI, Wei YANG, Wenhao YAN, Aisheng LIU, and Yanting TAN \\ Customer Service Center of the State Grid Corporation of China
}

\begin{abstract}
In the present scenario, continuous reforms in the social organization have become a new normal of maintaining the survival and development of enterprises and enhancing the competitiveness on the market, wherein the core of revolution is the change of management mode and leadership promotion. In this pursuit, the present study envisaged the innovation of organizational management mode of a subordinate secondary department of the Customer Service Center of the State Grid Corporation of China. Meanwhile, the revolution from the traditional bureaucratic organization to a novel platform-typed organization for the state-owned enterprises in internet evolution process was analyzed via the promotion of cadre leadership based on dual-requirements. The characteristic organization form with dual identities was built to better support the business development and compliance management, thereby achieving greater economic and social value creation for the enterprises.
\end{abstract}

Keywords. state-owned enterprises; Internet-based; Internet evolution; leadership; revolution of organization

\section{Introduction}

The revolution in a company organization is generally triggered by the adjustment of development strategy programming and evolves with the variations in the market and technological development. During the evolution of state-owned enterprises from the traditional bureaucratic organization to the internet-based platform organization, the devolution of the administrative power from the senior leaders to lower levels and the enhancement of leaderships of mid- and low-level cadres plays a key role in the success of the revolution of the organizational form and even the development strategy. The Customer Service Center of the State Grid Corporation of China (hereinafter referred to as the Customer Service Center or the Center) performs reconfiguration of the management factors, breaks the originally ossified bureaucracy management system, and establishes the platform-type organizations, with the aim of contributing to the enhancement of organization performance, ensuring high-speed business development, fast adaptation to ever-changing market changes and fast response to the customer requirements. 


\section{Design of lean management idea}

At present, the roots that shackle company development lie in the fact that the current organization structure cannot bear the internet-based development strategy, thereby failing in matching with the rapid iteration speed of the internet products. The employees, in particularly the introduced talents, face the contradiction between the traditional management requirements and the internet business development. They struggle to cope with the busy situation induced by mismatching between the job responsibilities and contents. Additionally, they are overburdened with work due to the stortage of staff and thereby cannot achieve their own values. On the other hand, the promotion channels are narrow, salary incentives are relatively weak and the personal development becomes impossible. In order to solve the contraction between the current rapid business development and the relatively weak organization, a task of top priority is to optimize both the organization and management system, and match and promote the productivity enhancement with more advanced productive relations. This will satisfy the requirements of the rapid business growth and achieve a sustained value creation.

In order to better adapt to the pulse of the market development and address a lot of problems, such as the mismatching between the internal management elements and the actual business requirements. The Customer Service Center of the State Grid Corporation of China, as a secondary administrative unit, investigated the internet enterprises such as Huawei[1] on the basis of originally traditional management mode of the state-owned enterprises. It also explored and merged into lean and agile management [2] thought of internet for guiding the overall design of the revolution. Moreover, under the background of supporting the construction of the new power system and achieving the combination of internet and customer service, the Customer Service Center broke the authoritarian leadership model, established the leadership rooted in high-, middle- and basic-level cadres, and builds a novel platform-typed organization (Figure 1 shows the house of leadership management ) that adopts the strategic goal of serving the customers and enhancing the customer satisfaction as core value pursuit. Based on the enterprise culture and values, the Customer Service Center laid an emphasis on the organization and human resource management, adopted communication as the link, respected employees' physical, intellectual, emotion security as well as their cultural requirements, and created a smooth working process along with a continuously improved working environment.

\section{Revolution of the organizational structure}

The most important thing in the core proposition of the organization development lies in the revolution of the organizational structure and the innovation in the talent mechanism. For the currently dispersive and variable market environment, the organizational structure should be flexible, so as to be compatible with the constant iteration and innovation of enterprises. This will become an important basis in the design of the platform-type organization.

In order to settle the problems like the mismatching between the organization mode and the business development degree, and the mismatching between the organization operating situation and rapidly iteratively market requirements, the secondary department explored the revolution in the organization structure on the basis of originally 4 departments and attempted the adjustments mainly in the following three aspects (Figure 2 shows the restructured organizational structure ). 


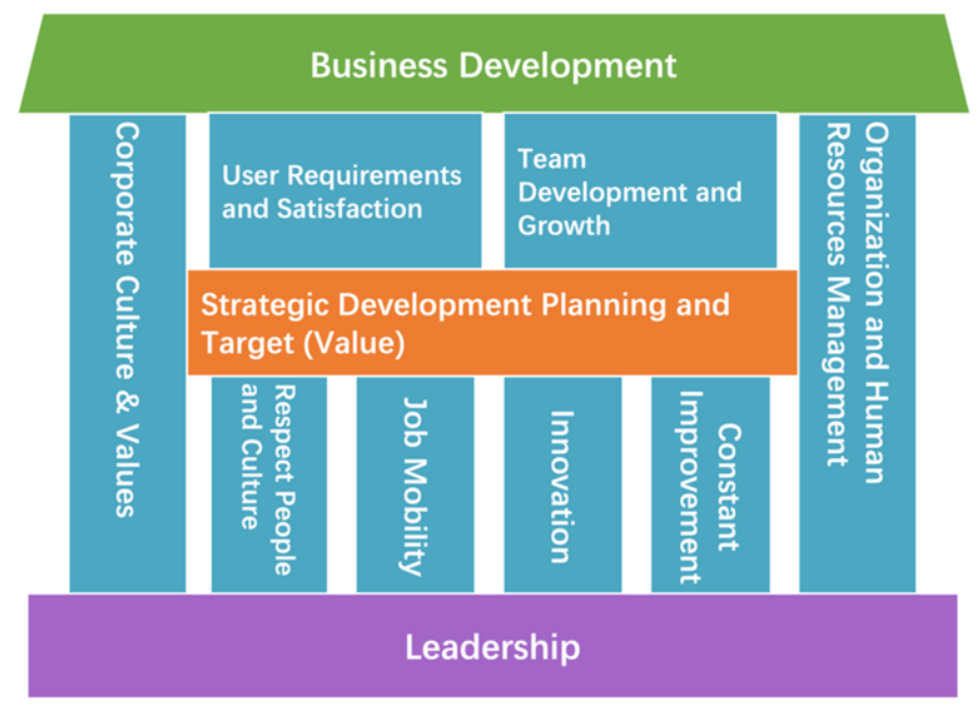

Figure 1. house of leadership management

Firstly, the general management department was created to the supporting platform with shared functions. The Customer Service Center focused on the business support and services, and compliance supervision, and built the business partner (BP) group that satisfied the front business requirements. Additionally, it provided the front BPs with specific incentive schemes for upgradation of the configuration, thereby injecting different degrees of resources for different projects and ensuring benefits and full operation to the full extent. Meanwhile, the Customer Service Center provided the management on the projects with injected resources after approval and achieved the business knowledge energization (mainly including strategy, finance and human resource) for the front-end.

Secondly, the vertical business unit was transformed into the empowered midground. Starting from the front-end requirements, the Customer Service Center integrates the precipitated resources and professional functions on the middle- and back-end, which could provide the modularized packing services and ability sinking services for the frontend organizations, thereby contributing to favorable performances on the front-end. In addition, performance management methods based on objective and key results (OKR) management was optimized by allocating enlightened and effective incentive policies and innovatively offering both inventive and energization mechanisms. The role of the mid-ground as the connector was released, so that the back-end resources could become the middlewares that could be called on demands, which energized both the professional resources on the front-end and professional knowledge with the delivery of middlewares. Meanwhile, the promotion channels for ordinary employees, technical experts, management and operation talents were built and both horizontal and vertical employee turnover mechanisms were set.

Thirdly, the front-end was elite. The Customer Service Center constructed three front-end business groups - ranger group, new business product group and traditional business product group. The customer requirements were scooped out continuously at multi-dimensions, so as to pull the organization by natural growth instincts at the front end. By reference to the organizational structure of agile development team, the product 
groups were created, so that all staff followed the command of users. Accordingly, the back-end functional departments that originally did not come into contact with the users were reconstructed, and the organizational form in which the front end was responsible for interacting with users and understanding the users' rigid demands. The mid-ground and the back-end actively organized various types of resources on the platform, so as to continuously output the products, services or solutions in good consistence with user demands, achieve the integration between flexible product groups/teams and rigid departments, and ensure full empowerment on the front end by the leadership. Meanwhile, the self-regulation on the front end was strengthened, so as to ensure highefficiency coordination among the various organizations on the front- and mid-grounds and ensure an effective establishment and operation of platform-typed organizations. Accordingly, the organization was not only a hierarchical organization, but also followed a moderately integrated and coherent-business organizational operating mode.

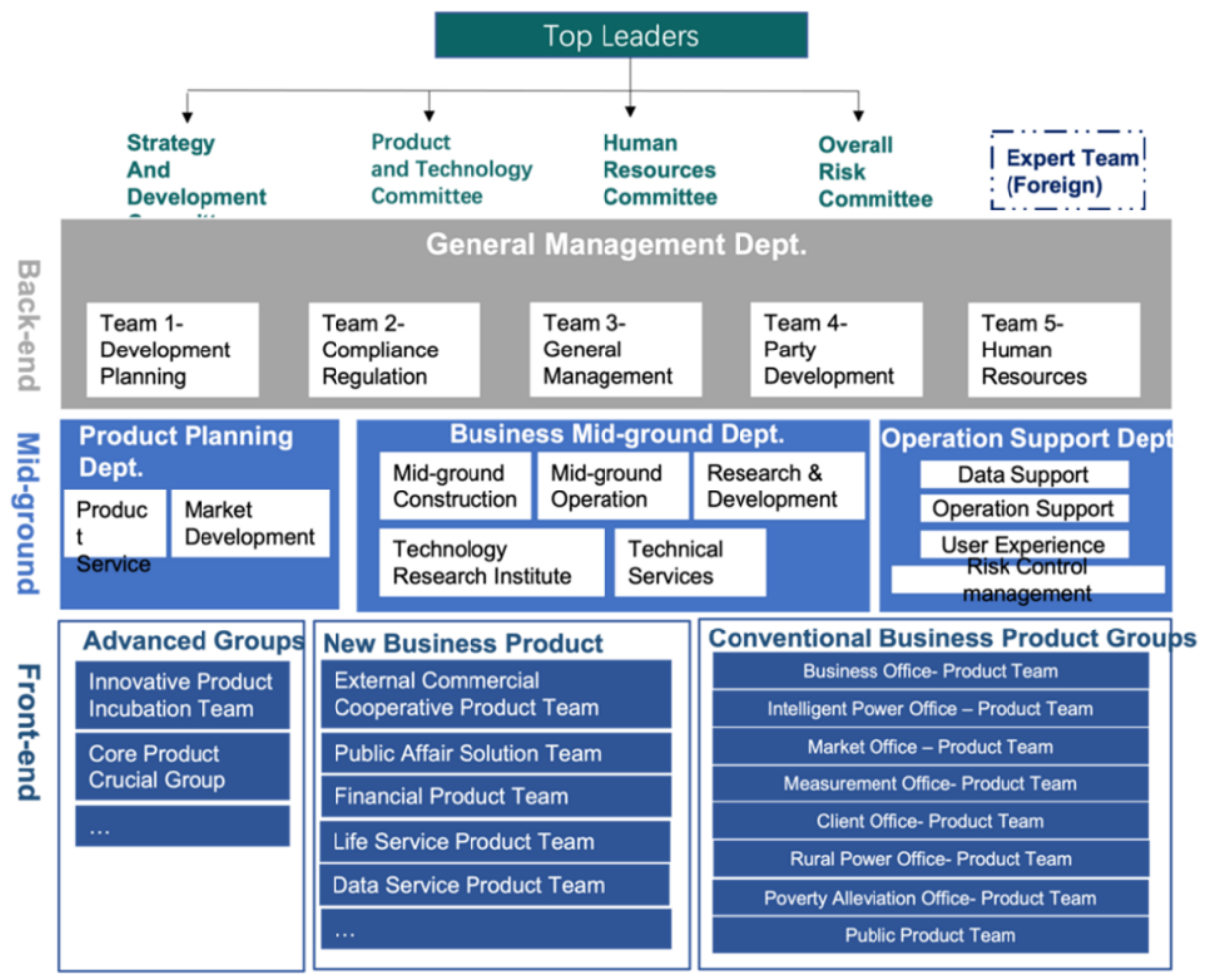

Figure 2. organizational structure diagram

During the overall organizational revolution process, the Customer Service Center obeyed the principle of small steps for running and continuous iteration, performed the adjustment of organization structures and gradually increased and adjusted the personnel. In accordance with the principle of inclination towards the front-line and healthy competition, the Customer Service Center gave the priority to the allocation towards the product groups, attempted resource allocation and dynamic adjustment within the product groups, and encouraged to preferentially allocate advantageous resources for advantageous products. In additionally, based on the principle of talent pooling and 
resource coordination, the outstanding employees with leadership were encouraged, who were allocated to new posts or product groups for training, so as to reserve high-quality versatile talents for organizations.

\section{Leadership promotion}

An organization is composed of people. People are the important factor that achieves the organizational goal. A series of problems were encountered, including the mismatching of the current management mechanism and promotion channel with the personal development, professional posts and actual work requirements of personnels, the mismatching between the reserve of talents and business development, and the mismatching of performance management with the diversified positions and personnel management requirements. In order to solve these problems, the secondary department strived to achieve a synchronous revolution of the management and leadership, released the management functions of the middle- and basic-level leading cadres and broke the ceiling of enterprise development. By setting talent selection as the gripper that grasped the driving source of development, the department stimulated multi-level needs of people via talent selection, drove away the laziness in human nature and leveraged a greater value creation through reasonable distribution of rights.

The department main considered the revolution in the following three aspects: Firstly, the leader level clearly defined the keynote of self-targeted revolution, changed both the leadership concept and style, made the transition from command leadership to service leadership, promoted the fault-tolerant spirit, and supported and energized the team innovation. Meanwhile, the department constructed four-vertical, six-horizontal and three-qualified post rank sequences, helped the employees for careering planning and guidance in accordance with their own characteristics and overall enhanced both the leadership and professional capabilities of employees. Accordingly, the organizational ability was effectively enhanced, so as to achieve win-win results of employee career development and enterprise management development. Secondly, the thought of agile values was publicized by the senior leaders, systematically arranged, dismantled and implemented by the mid- and basic-level leaders. By creating pilot product groups and forming benchmarking cases, rapid launching and validation of products was combined with material and immaterial incentives for team, thereby effectively stimulating the working and innovation enthusiasms of team. Thirdly, by reference to personnel capability evaluation and grading modes in internet companies, the key performance indicators (KPIs) were set in combination with objectives and key results (OKR) according to the department management requirements, and OKR indexes were set in combination with product innovation. Moreover, the corresponding performance assessment tool was designed, so as to assist strategic conduction, hierarchical classification and effective revolution of management mode, which provided a multidimensional development opportunities and growth space for talents. At the same time, the internal capital appreciation cycles featured by value creation, value evaluation and value distribution were re-constructed, while a just, fair and open healthy comparison, and a competition environment was built to achieve self-leading and self-driving of employees. This further contributed to the organizational self-operation. 


\section{Conclusion}

In the present study, the secondary department under the administration of the Customer Service Center first analyzed and clarified the development strategic positioning and development mode in depth, and performed the research on platform-type organization revolution. By diagnosing and analyzing the problems in the implementation of matrix agile organizations in transverse process flow, the platform-type organizational mode featured by resource back-end, empowered mid-ground, custom front-end was investigated in combination with actual development and business requirements, and simultaneously, a novel organization management model and the supporting measures and mechanisms were constructed. After the selection and establishment of the institutional reforms and flexible teams, an organizational form abundant in novel leadership with diversified management and agile products was successfully built. The leading cadres at different levels could communicate and coordinate with employees, so that the development strategy could be decomposed and implemented step by step. The department also made innovations in the management and motivation modes, and encouraged the employees to work actively for achieving the organization's development goal. This ensured a good integration and mutual promotion between the management requirements of the state-owned enterprises and the development characteristics of internet.

\section{References}

[1] Tian Tao, David De Cremer and Wu Chunbo,.Huawei: Leadership, Culture and Connectivity,.New Delhi: SAGE Publications; 2018. 424p.

[2] Dean Leffingwell. SAFe 4.0 Reference Guide: Scaled Agile Framework for Lean Software and Systems Engineering. China Machine Press; 2017. 356p. 